\title{
Phase diagram of a class of spin-triplet ferromagnetic superconductors
}

\author{
D. V. Shopova* and D. I. Uzunov \\ CPCM Laboratory, G. Nadjakov Institute of Solid State Physics, \\ Bulgarian Academy of Sciences, BG-1784 Sofia, Bulgaria. \\ * Corresponding author: sho@issp.bas.bg
}

Key words: superconductivity, ferromagnetism, phase diagram, order parameter profile.

PACS: 74.20.De, 74.20.Rp

\begin{abstract}
We investigate thermodynamic phases, including the phase of coexistence of superconductivity and ferromagnetism, the possible phase transitions of first and second order, and the shape of the phase diagram in mean-field approximation for a phenomenological model of spin-triplet ferromagnetic superconductors. The results are discussed in view of application to metallic ferromagnets as $\mathrm{UGe}_{2}, \mathrm{ZrZn}_{2}$, URhGe, and Fe.
\end{abstract}

Recently, the coexistence of ferromagnetism and superconductivity was discovered in the metallic compounds $\mathrm{UGe}_{2}$ [1, 2, 3], $\mathrm{ZrZn}_{2}$ [4], $\mathrm{URhGe}$ [5] and also in Fe [6] in experiments at low temperatures and high pressure. In contrast to other superconducting materials, in these metals the phase transition temperature to the ferromagnetic state is higher than the phase transition temperature to the superconducting state. Moreover, it seems that the superconductivity in the metallic compounds mentioned above always coexists with the ferromagnetic order and is enhanced by the latter. In these systems the superconductivity seems to arise from the same electrons that create the band magnetism, and is most naturally understood as a triplet rather than spin-singlet pairing phenomenon [7, 8, 9]. The same unconventional superconductivity has been suggested [1] as a possible outcome of the interpretation of experiments in Fe [6]. Note, that both vortex and Meissner superconductivity phases [6] are found in the high-pressure crystal modification of Fe which has a hexagonal close-packed lattice. In this hexagonal lattice the strong ferromagnetism of the usual bcc iron crystal probably disappears [10].

Recently, the phenomenological theory that explains coexistence of ferromagnetism and unconventional spin-triplet superconductivity of Landau-Ginzburg type was developed 
[11, 12. The possible low-order coupling between the superconducting and ferromagnetic order parameters is derived on the basis of general symmetry group arguments and several important features of the superconducting vortex state in the ferromagnetic phase of unconventional ferromagnetic superconductors are established [11, 12].

In this letter we shall use the approach presented in Refs. [11, 12, to investigate the conditions for the occurrence of the Meissner phase and to demonstrate that the presence of ferromagnetic order enhances the $p$-wave superconductivity. For this aim we shall establish the phase diagram corresponding to model ferromagnetic superconductors in a zero external magnetic field. We shall also show that the phase transition to the superconducting state in ferromagnetic superconductors can be either of first or second order depending on the particular substance. We confirm the predictions made in Refs. [11, 12] about the symmetry of the ordered phases.

Our investigation is based on the mean-field approximation [13] as well as on familiar results about the possible phases in nonmagnetic superconductors with triplet ( $p$-wave) Cooper pairs 17, 14, 15]. We shall neglect all anisotropies, usually given by the respective additional Landau invariants and gradient terms [8, 9] in the Ginzburg-Landau free energy of unconventional superconductors. The reasons is that the inclusion of crystal anisotropy is related with lengthy formulae and a multivariant analysis which will obscure our main aims and results. Let us emphasize that the present results should be valid in the same or modified form when the crystal anisotropy is properly taken into account. We have to mention also that there is a formal similarity between the phase diagram obtained in our investigation and the phase diagram of certain improper ferroelectrics [16, 17.

Following Refs. 11, 12] we consider the Ginzburg- Landau free energy $F=\int d^{3} x f(\psi, \overrightarrow{\mathcal{M}})$, where

$$
f=\frac{\hbar^{2}}{4 m}\left(D_{j}^{*} \psi\right)\left(D_{j} \psi\right)+a_{s}|\psi|^{2}+\frac{b}{2}|\psi|^{4}+a_{f} \overrightarrow{\mathcal{M}}^{2}+\frac{\beta}{2} \mathcal{M}^{4}+i \gamma_{0} \overrightarrow{\mathcal{M}} \cdot\left(\psi \times \psi^{*}\right) .
$$

In Eq. (11), $D_{j}=\left(\nabla-2 i e A_{j} / \hbar c\right)$, where $A_{j}(j=1,2,3)$ are the components of the vector potential $\vec{A}$ related with the magnetic induction $\vec{B}=\nabla \times \vec{A}$, the complex vector $\psi=\left\{\psi_{j}\right\} \equiv\left(\psi_{1}, \psi_{2}, \psi_{3}\right)$ is the superconducting order parameter, corresponding to the spin-triplet Cooper pairing and $\overrightarrow{\mathcal{M}}=\left\{\mathcal{M}_{j}\right\}$ is the magnetization. The coupling constant $\gamma_{0}=4 \pi J>0$ is given by the ferromagnetic exchange parameter $(J>0)$. Coefficients $a_{s}=\alpha_{s}\left(T-T_{s}\right)$ and $a_{f}=\alpha_{f}\left(T-T_{f}\right)$ are expressed by the positive parameters $\alpha_{s}$ and $\alpha_{f}$ as well as by the superconducting $\left(T_{s}\right)$ and ferromagnetic $\left(T_{f}\right)$ critical temperatures in the decoupled case, when $\mathcal{M} \psi$-interaction is ignored; $b>0$ and $\beta>0$ as usual. Note, that all seven material parameters $\left(\alpha_{s}, \alpha_{f}, T_{s}, T_{s}, b, \beta, J\right)$ depend on material properties, the temperature $T$ and additional intensive thermodynamic parameters as pressure $P$.

We assume that the magnetization $\mathcal{M}$ is uniform, which is a reliable assumption outside a quite close vicinity of the magnetic phase transition whereas we keep the spatial $(\vec{x}-)$ dependence of $\psi$. The reason is that the relevant dependence of $\psi$ on $\vec{x}$ is generated by the diamagnetic effects arising from the presence of $\mathcal{M}$ and the external magnetic field 
$\vec{H}$ [11, 12] rather than from fluctuations of $\psi$ (this effect is extremely small and can be safely ignored). Note, that the first term in (11) still persists for $\vec{H}=0$ because of the diamagnetic effect created by of magnetization $\overrightarrow{\mathcal{M}}=\vec{B} / 4 \pi>0$. As we shall investigate the conditions for the occurrence of the Meissner phase where $\psi$ is uniform, the spatial dependence of $\psi$ and, hence, the first term in r.h.s. of (11) will be neglected (see also a brief discussion at the end of this paper).

In case of a strong easy axis type of magnetic anisotropy, as is in $\mathrm{UGe}_{2}$ [1], the overall complexity of mean-field analysis of the free energy (11) can be avoided by performing an Ising-like description: $\overrightarrow{\mathcal{M}}=(0,0, \mathcal{M})$, where $\mathcal{M}= \pm|\mathcal{M}|$ is the magnetization along the "z-axis." Further, because of the equivalence of the two physical states $( \pm \mathcal{M})$ the thermodynamic analysis can be performed within the "gauge" $\mathcal{M} \geq 0$. But this stage of consideration can also be achieved without the help of crystal anisotropy arguments. When the magnetic order has a continuous symmetry one may take advantage of the symmetry of model (10) and avoid the consideration of equivalent thermodynamic states that occur as a result of the respective symmetry breaking at the phase transition point but have no effect on thermodynamics of the system. In the isotropic system one may again choose a gauge, in which the magnetization vector has the same direction as $z$-axis $\left(|\overrightarrow{\mathcal{M}}|=\mathcal{M}_{z} \equiv \mathcal{M}\right)$ and this will not influence the generality of thermodynamic analysis.

With the help of convenient notations, $\varphi_{j}=b^{1 / 4} \psi_{j}, \varphi_{j}=\phi_{j} \exp \left(\theta_{j}\right), M=\beta^{1 / 4} \mathcal{M}, \gamma=$ $\gamma_{0} /\left(b^{2} \beta\right)^{1 / 4}, r=a_{s} / \sqrt{b}, t=a_{f} / \sqrt{\beta}$, and neglecting the first term in (11) the free energy becomes

$$
f=r|\phi|^{2}+\frac{1}{2}|\phi|^{4}+t M^{2}+\frac{1}{2} M^{4}+2 \gamma M \phi_{1} \phi_{2} \sin \theta
$$

where $\phi^{2}=\left(\phi_{1}^{2}+\phi_{2}^{2}+\phi_{3}^{2}\right)$, and $\theta=\left(\theta_{2}-\theta_{1}\right)$.

The possible (stable, metastable and unstable) phases are given in Table 1 together with the respective existence and stability conditions. The stability conditions define the domain of the phase diagram where the respective phase is either stable or metastable [13. The normal (disordered) phase, denoted in Table 1 by $N$ always exists (for all temperatures $T \geq 0$ ) but is stable for $t>0, r>0$. The superconductivity phase denoted in Table 1 by $\mathrm{SC} 1$ is unstable. The same is valid for the phase of coexistence of ferromagnetism and superconductivity denoted in Table 1 by CO2. The N-phase, the ferromagnetic phase (FM), the superconducting phases (SC1-3) and two of the phases of coexistence (CO1-3) are generic phases because they appear also in the decoupled case $(\gamma \equiv 0)$. When the $M \psi$-coupling is not present, the phases SC1-3 are identical and represented by the order parameter $\varphi$ where the components $\varphi_{j}$ participate on equal footing. The asterisk attached to the stability condition of "the second superconductivity phase", (SC2), indicates that our analysis is insufficient to determine whether this phase corresponds to a minimum of the free energy. As we shall see later the phase SC2, as well as the other two purely superconducting phases and the coexistence phase CO1, have no chance to become stable for $\gamma \neq 0$. This is so, because the non-generic phase of coexistence of superconductivity and ferromagnetism (FS in Table 1), which does not exist for $\gamma=0$ is stable and has a lower free energy in their domain of stability. 
TABLE 1 . Phases and their existence and stability properties $(k=0, \pm 1, \ldots)$.

\begin{tabular}{|l|l|l|l|}
\hline \hline Phase & order parameter & existence & stability domain \\
\hline $\mathrm{N}$ & $\phi_{j}=M=0$ & always & $t>0, r>0$ \\
\hline $\mathrm{FM}$ & $\phi_{j}=0, M^{2}=-t$ & $t<0$ & $r>0, r^{2}>\gamma^{2} t$ \\
\hline $\mathrm{SC} 1$ & $\phi_{1}=M=0, \phi^{2}=-r$ & $r<0$ & unstable \\
\hline $\mathrm{SC} 2$ & $\phi^{2}=-r, \theta=\pi k, M=0$ & $r<0$ & $(t>0)^{*}$ \\
\hline $\mathrm{SC} 3$ & $\phi_{1}=\phi_{2}=M=0, \phi_{3}^{2}=-r$ & $r<0$ & $r<0, t>0$ \\
\hline $\mathrm{CO} 1$ & $\phi_{1}=\phi_{2}=0, \phi_{3}^{2}=-r, M^{2}=-t$ & $r<0, t<0$ & $r<0, t<0$ \\
\hline $\mathrm{CO} 2$ & $\phi_{1}=0, \phi^{2}=-r, \theta=\theta_{2}=\pi k, M^{2}=-t$ & $r<0, t<0$ & unstable \\
\hline $\mathrm{FS}$ & $2 \phi_{1}^{2}=2 \phi_{2}^{2}=\phi^{2}=-r+\gamma M, \phi_{3}=0$ & $\gamma M>r$ & $3 M^{2}>\left(-t+\gamma^{2} / 2\right)$ \\
& $\theta=2 \pi(k-1 / 4), \gamma r=\left(\gamma^{2}-2 t\right) M-2 M^{3}$ & & $M>0$ \\
\hline \hline
\end{tabular}

We have outlined the domain in the $(t, r)$ plane where the FS phase exists and is a minimum of the free energy. For $r<0$ the third-degree algebraic equation for $M$ (see Table 1) and the existence and stability conditions are satisfied for any $M \geq 0$ provided $t \geq \gamma^{2}$. For $t<\gamma^{2}$ the condition $M \geq M_{0}$ have to be fulfilled, here the value $M_{0}=\left(-t+\gamma^{2} / 2\right)^{1 / 2}$ of $M$ is obtained from $r\left(M_{0}\right)=0$. Thus for $r=0$ the $\mathrm{N}$-phase is stable for $t \geq \gamma^{2} / 2$, on the other hand FS is stable for $t \leq \gamma^{2} / 2$. For $r>0$, the requirement for the stability of FS leads to the inequalities

$$
\max \left(\frac{r}{\gamma}, M_{m}\right)<M<M_{0},
$$

where $M_{m}=\left(M_{0} / \sqrt{3}\right)$ and $M_{0}$ should be the positive solution of the third-degree equation of state from Table $1 ; M_{m}>0$ gives a maximum of the function $r(M)$.

The further analysis leads to the existence and stability domain of FS below the line AB given by circles (see Fig. 1). In Fig. 1 the curve of circles starts from the point A with coordinates $\left(\gamma^{2} / 2,0\right)$ and touches two other (solid and dotted) curves at the point B with coordinates $\left(-\gamma^{2} / 4, \gamma^{2} / 2\right)$. Line of circles represents the function $r\left(M_{m}\right) \equiv r_{m}(t)$ where

$$
r_{m}(t)=\frac{4}{3 \sqrt{3} \gamma}\left(\frac{\gamma^{2}}{2}-t\right)^{3 / 2}
$$

Dotted line is given by $r_{e}(t)=\gamma \sqrt{|t|}$. The inequality $r<r_{m}(t)$ is a condition for the stability of FS, whereas the inequality $r \leq r_{e}(t)$ for $(-t) \geq \gamma^{2} / 4$ is a condition for the existence of FS as a solution of the respective equation of state. This existence condition for FS has been obtained from $\gamma M>r$ (see Table 1 ).

In the region on the left of the point B in Fig. 1, the FS phase satisfies the existence condition $\gamma M>r$ only below the dotted line. In the domain confined between the lines of circles and the dotted line on the left of the point B the stability condition for FS is satisfied but the existence condition is broken. The inequality $r \geq r_{e}(t)$ is the stability condition of FM for $0 \leq(-t) \leq \gamma^{2} / 4$. For $(-t)>\gamma^{2} / 4$ the FM phase is stable for all $r \geq r_{e}(t)$. 


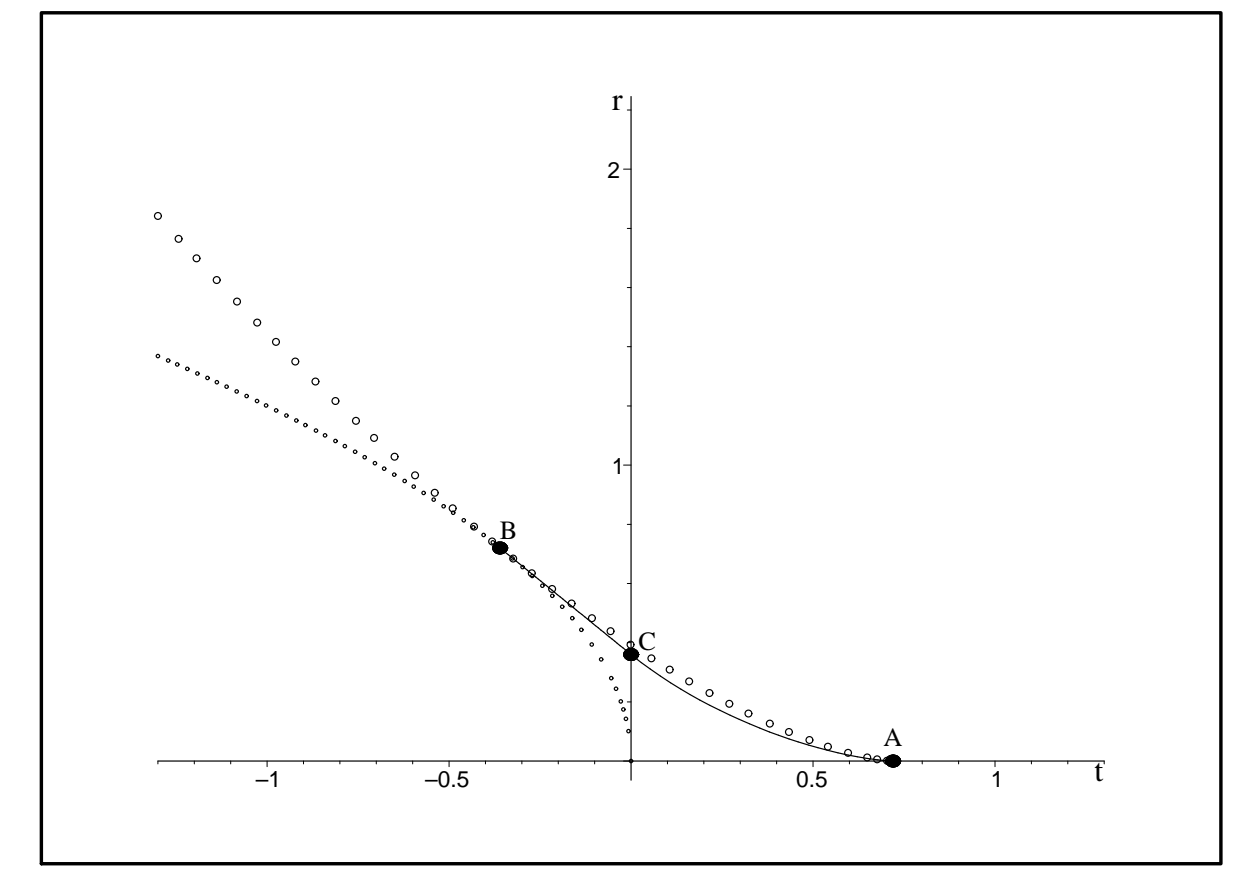

Figure 1: The phase diagram in the plane $(t, r)$ with two tricritical points $(\mathrm{A}$ and $\mathrm{B})$ and a triple point $C ; \gamma=1.2$.

In the region confined by the line of circles $\mathrm{AB}$, the dotted line for $0<(-t)<\gamma^{2} / 4$, and the $t$-axis, the phases N, FS and FM have an overlap of stability domains. The same problem exists for FS and the SC phases in the second quadrant and for the phases FS and CO1 in the third quadrant of the plane $(t, r)$. The comparison of the respective free energies for $r<0$ shows that the stable phase is FS whereas the other phases are metastable within their domains of stability.

The part of the $t$-axis given by $r=0$ and $t>\gamma^{2} / 2$ is a phase transition line of second order which describes the N-FS transition. The same transition for $0<t<\gamma^{2} / 2$ is represented by the solid line $\mathrm{AC}$ which is the equilibrium transition line of a first order phase transition. This equilibrium transition curve is given by the function

$$
\left.r_{e q}(t)=\frac{1}{4}\left[3 \gamma-\left(\gamma^{2}+16 t\right)\right)^{1 / 2}\right] M_{e q}(t)
$$

where

$$
M_{e q}(t)=\frac{1}{2 \sqrt{2}}\left[\gamma^{2}-8 t+\gamma\left(\gamma^{2}+16 t\right)^{1 / 2}\right]^{1 / 2}
$$

is the equilibrium value (jump) of the magnetization. The order of the N-FS transition changes at the tricritical point A.

The domain above the solid line AC and below the line of circles for $t>0$ is the region of a possible overheating of FS. The domain of overcooling of the N-phase is confined by the solid line $\mathrm{AC}$ and the axes $(t>0, r>0)$. At the triple point $\mathrm{C}$ with coordinates $[0$, 
$\left.r_{e q}(0)=\gamma^{2} / 4\right]$ the phases N, FM, and FS coexist. For $t<0$ the straight line

$$
r_{e q}^{*}(t)=\frac{\gamma^{2}}{4}+|t|, \quad-\gamma^{2} / 4<t<0,
$$

describes the extension of the equilibrium phase transition line of the N-FS first order transition to negative values of $t$. For $t<\left(-\gamma^{2} / 4\right)$ the equilibrium phase transition FMFS is of second order and is given by the dotted line on the left of the point $\mathrm{B}$ (the second tricritical point in this phase diagram). Along the first order transition line $r_{e q}^{*}(t)$ given by (8) the equilibrium value of $M$ is $M_{e q}=\gamma / 2$, which implies an equilibrium order parameter jump at the FM-FS transition equal to $(\gamma / 2-\sqrt{|t|})$. On the dotted line of the second order FM-FS transition the equilibrium value of $M$ is equal to that of the FM phase $\left(M_{e q}=\sqrt{|t|}\right)$. Note, that the FS phase does not exists below $T_{s}$ and this seems to be a disadvantage of the model (11).

The equilibrium phase transition lines of the FM-FS and N-FS transition lines in Fig. 1 can be expressed by the respective equilibrium phase transition temperatures $T_{e q}$ defined by the equations $r_{e}=r\left(T_{e q}\right), r_{e q}=r\left(T_{e q}\right), r_{e q}^{*}=r\left(T_{e q}\right)$, and with the help of the relation $M_{e q}=M\left(T_{e q}\right)$. This leads to some limitations on the possible variations of the parameters of the theory. For example, the critical temperature $\left(T_{e q} \equiv T_{c}\right)$ of the FM-FS transition of second order $\left(\gamma^{2} / 4<-t\right)$ is obtained in the form $T_{c}=\left(T_{s}+4 \pi J \mathcal{M} / \alpha_{s}\right)$, or, using $\mathcal{M}=\left(-a_{f} / \beta\right)^{1 / 2}$,

$$
T_{c}=T_{s}-\frac{T^{*}}{2}+\left[\left(\frac{T^{*}}{2}\right)^{2}+T^{*}\left(T_{f}-T_{s}\right)\right]^{1 / 2},
$$

where $T_{f}>T_{s}$, and $T^{*}=(4 \pi J)^{2} \alpha_{f} / \alpha_{s}^{2} \beta$ is a characteristic temperature of the model (II). The investigation of the conditions for the validity of (8) leads to the conclusion that the FM-FS continuous phase transition (at $\gamma^{2}<-t$ ) is possible only if the following condition is satisfied:

$$
T_{f}-T_{s}>=(\varsigma+\sqrt{\varsigma}) T^{*},
$$

where $\varsigma=\beta \alpha_{s}^{2} / 4 b \alpha_{f}^{2}$. This means that the second order FM-FS transition should disappear for sufficiently large $M \psi$-coupling. Such a condition does not exist for the first order transitions FM-FS and N-FS.

Taking into account the first term in the free energy (11) should lead to a depression of the equilibrium transition temperature. As the magnetization increases with the decrease of the temperature, the vortex state should occur at temperatures which are lower than the equilibrium temperature $T_{e q}$ of the homogeneous (Meissner) state. For example, the critical temperature $\left(\tilde{T}_{c}\right)$ corresponding to the inhomogeneous (vortex) phase of FS-type has been evaluated [12] to be lower than the critical temperature (8): $\left(T_{c}-\tilde{T}_{c}\right)=$ $4 \pi \mu_{B} \mathcal{M} / \alpha_{s}\left(\mu_{B}=|e| \hbar / 2 m c\right.$ - Bohr magneton). For $J \gg \mu_{B}$, we have $T_{c} \approx \tilde{T}_{c}$. Finally, let us emphasize that a more reliable description of these phenomena, in particular, of the thermodynamic behaviour of the FS phase at relatively large values of $\mathcal{M}$ can be performed if an additional term of type $\mathcal{M}^{2}|\psi|^{2}$ is included in the model (11). 
Acknowledgments: DIU thanks the hospitality of MPI-PKS-Dresden. Financial support through Scenet (Parma) and collaborative project with JINR-Dubna is also acknowledged. 


\section{References}

[1] S. S. Saxena, P. Agarwal, K. Ahilan, F. M. Grosche, R. K. W. Haselwimmer, M.J. Steiner, E. Pugh, I. R. Walker, S.R. Julian, P. Monthoux, G. G. Lonzarich, A. Huxley. I. Sheikin, D. Braithwaite, and J. Flouquet, Nature (London) 406 (2000) 587.

[2] A. Huxley, I. Sheikin, E. Ressouche, N. Kernavanois, D. Braithwaite, R. Calemczuk, and J. Flouquet, Phys. Rev. B 63 (2001) 144519-1.

[3] N. Tateiwa, T. C. Kobayashi, K. Hanazono, A. Amaya, Y. Haga. R. Settai, and Y. Onuki, J. Phys. Condensed Matter, 13 (2001) L17.

[4] C. Pfleiderer, M. Uhlatz, S. M. Hayden, R. Vollmer, H. v. Löhneysen, N. R. Berhoeft, and G. G. Lonzarich, Nature (London) 412 (2001) 58.

[5] D. Aoki, A. Huxley, E. Ressouche, D. Braithwaite, J. Flouquet, J-P.. Brison, E. Lhotel, and C. Paulsen, Nature (London) 413 (2001) 613.

[6] K. Shimizu, T. Kimura, S. Furomoto, K. Takeda, K. Kontani, Y. Onuki and K. Amaya, Nature (London) 412 (2001) 316.

[7] G. E. Volovik and L. P. Gor'kov, Sov. Phys. JETP 61 (1985) 843 [Zh. Eksp. Teor. Fiz. 88 (1985) 1412].

[8] M. Sigrist and K. Ueda, Rev. Mod. Phys. 63 (1991) 239.

[9] V. P. Mineev, K. V. Samokhin, Introduction to Unconventional Superconductivity (Gordon and Breach, Amsterdam, 1999).

[10] S. S. Saxena and P. B. Littlewood, Nature (London) 412 (2001) 290.

[11] K. Machida and T. Ohmi, Phys. Rev. Lett. 86 (2001) 850.

[12] M. B. Walker and K. V. Samokhin, Phys. Rev. Lett. 88 (2002) 204001-1.

[13] D. I. Uzunov, Theory of Critical Phenomena (World Scientific, Singapore, 1993).

[14] E. J. Blagoeva, G. Busiello, L. De Cesare, Y. T. Millev, I. Rabuffo, and D. I. Uzunov, Phys. Rev. B 40 (1990) 7321.

[15] D. I. Uzunov, in: Advances in Theoretical Physics, ed. by E. Caianiello (World Scientific, Singapore, 1990) p. 96.

[16] Yu. M. Gufan and V. I. Torgashev, Sov. Phys. Solid State 22 (1980) 951 [Fiz. Tv. Tela (Leningrad) 22 (1980) 1629].

[17] Yu. M. Gufan, E. I. Kut'in, V. L. Lorman, and E. N. Sidorenko, Sov. Phys. Solid State 29 (1987) 432 [Fiz. Tv. Tela (Leningrad) 29 (1986) 756]. 